\title{
دراسة حقلية لتأثير النفط المتسرب في بعض العوامل البيئية وتقديرها في المياه
}

\author{
علي مهدي ناصر \\ فارس جاسم محمد الامارة
}

قسم الكيمياء البيئية البحرية ـ مركز علوم البحار - جامعة البصرة ـ البصرة ـ العراق

\section{الخلاصة}

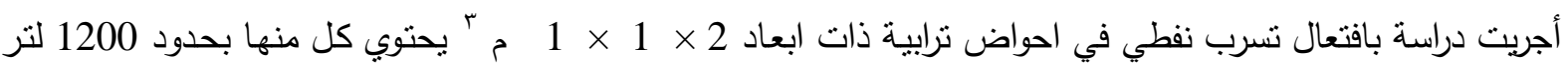
من مياه نهر كرمة علي, اضيف للاحواض نفط خام البصرة بواقع 1.2 لنر للأول ه

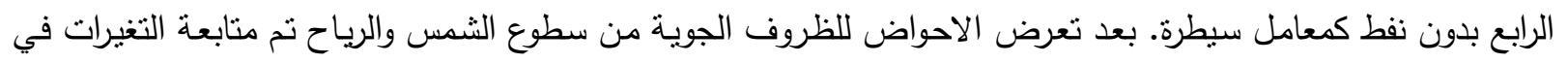

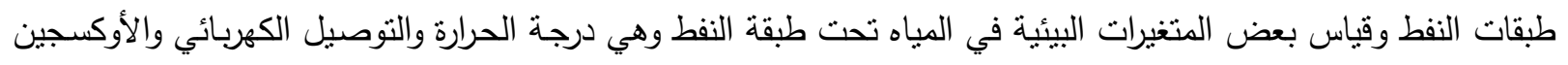

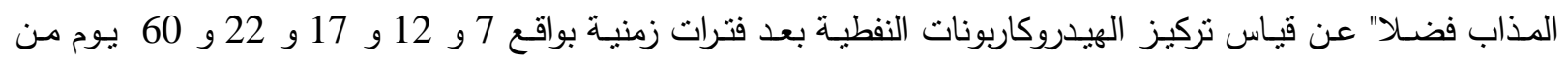

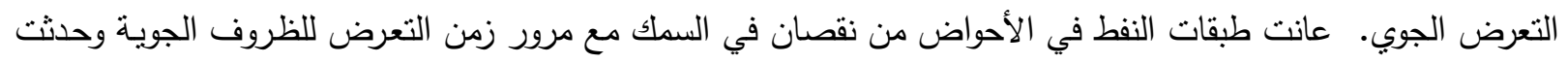

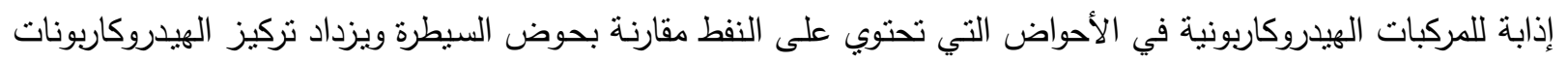

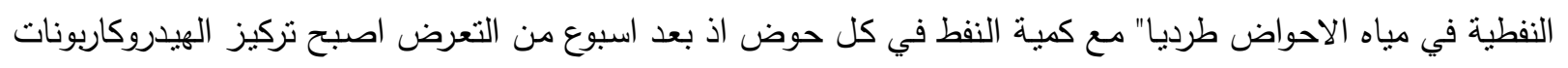

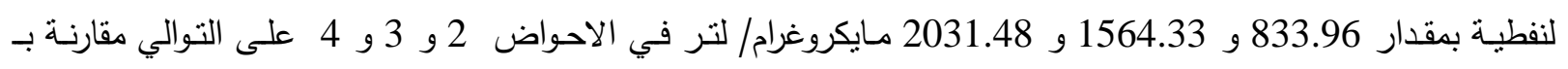

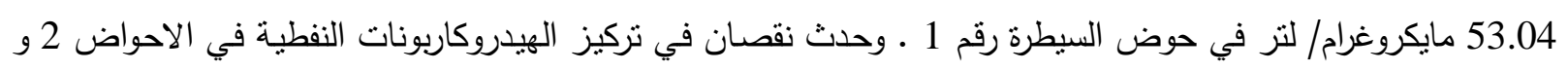

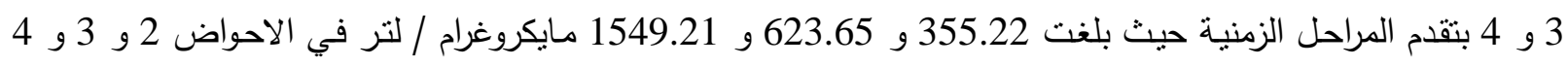

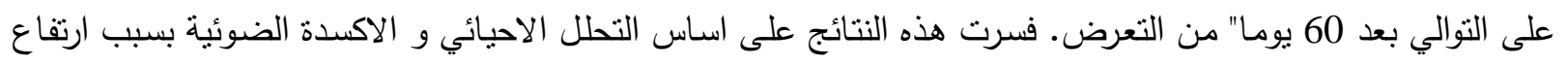

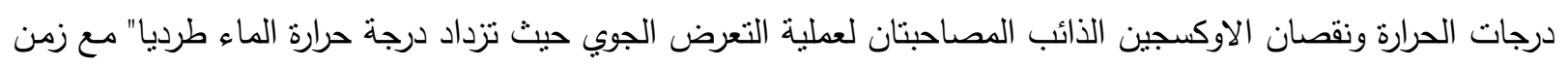

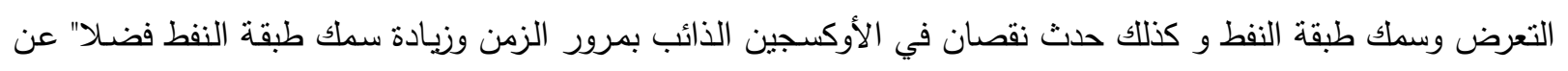

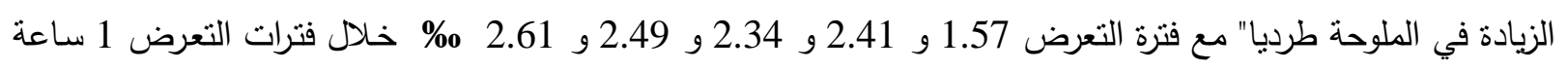

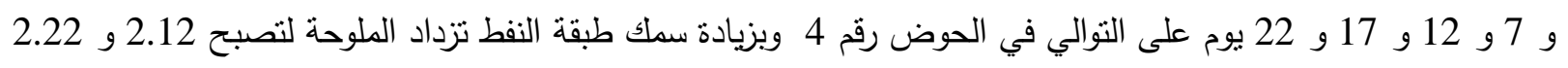
و 2.61\% في الأحواض 2 و 3 و و 4 على التوالي بعد 22 يوم من من التعرض للظروف الجوية . 
(Payne \&Phillips, التحلل الكيميائي المقدمة

1985; Berthou, et al., 1985) للهيدروكاربونات النفطية في الطبقة السطحية للنفط المتسرب تكمن في تقدير وتقييم الدكونات الكيميائية

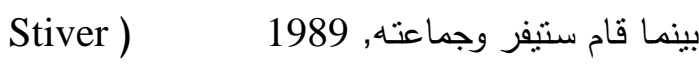
(et al., 1989 معينة من الهيدروكاربونات في النفط الخام. الهدف من هذه الدراسة

دراسة مصير النفط المنسرب في اليياه العذبة

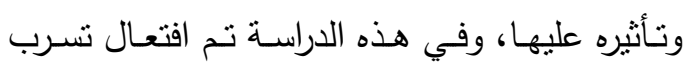

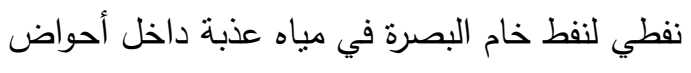

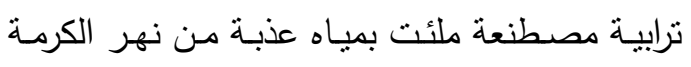

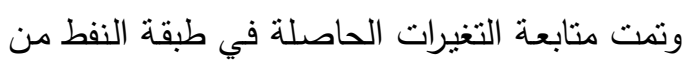
خـلال مثابعـة التغيـرات في المواصـفات الكيميائيـة والفيزيائية في طبقة المباه التي تحتها.

\section{المواد وطرائق العمل تصميم التجرية}

ن تصميم اربعة احواض ترابية بحجم 21 X 2

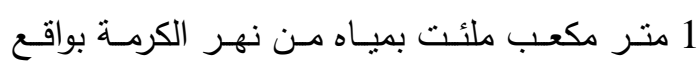
1200 لنر للحوض الواحد. اضيف للاحواض 2 و 3

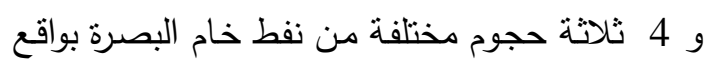

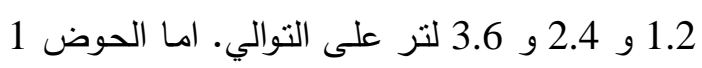

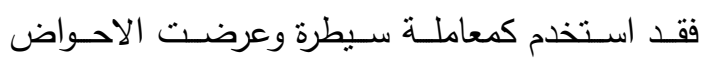
الاربعة للظروف الجوية الطبيعية ( الثكل 1 ).
يعتبر النفط الخام ومشتقاته من بين الملوثات

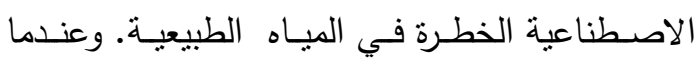

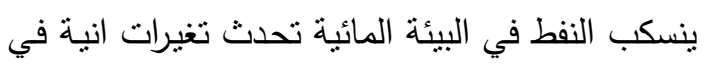

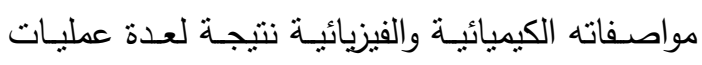
Blumer et al., 1973 ; Lee et (ونوية متزامنة

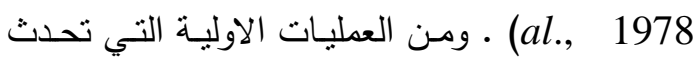

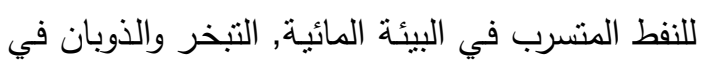
عمود الماء واختراق ترب قعر الممر المائي والاكسدة

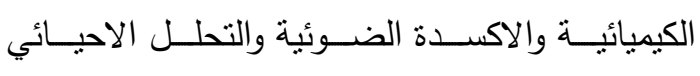
(NAS,1985)

النفط مزيج معقد من المركبات الهيدروكاربونية من بينها مركبات صغيرة التركيب وسريعة النطاير

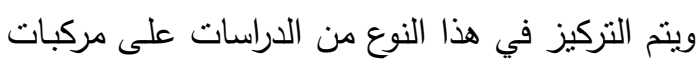

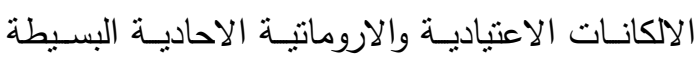

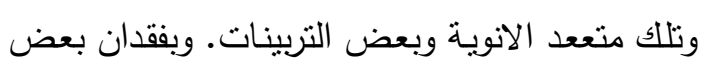
الهيدروكاربونات بسبب التجوية يتغير مكونات النفط, وتكمن الخطورة العظمى للبيئة المائية في المركبات

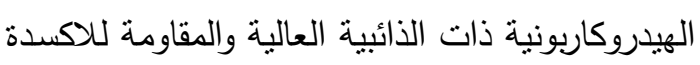
والتـي يمكن ان تصـل تراكيزهـا بحدود 0.1 - 0.5 مللي غراج/لتز (Mikhilova,1987). خلال العقود الاربعة الاخيرة من القرن العشرين

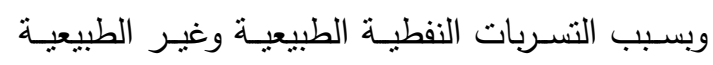

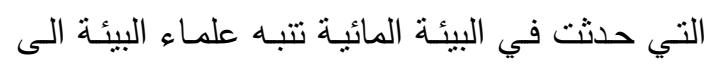

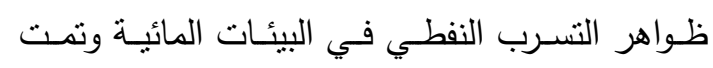
متابعة مصير النفوط المنسكبة ومعظم هذه الدراسات تم تتفيذها في المختبرات ( Gordon et al., 1976 (Hanson, 1975; Berthou et al., 1985 (Mackay et al., 1981 Blumer وكنلك حقلياً et al., 1973; DouAbul and Al-Saadi,

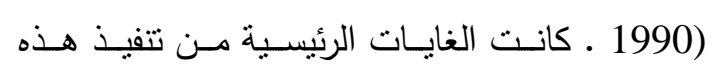

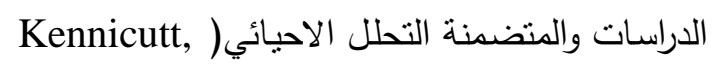




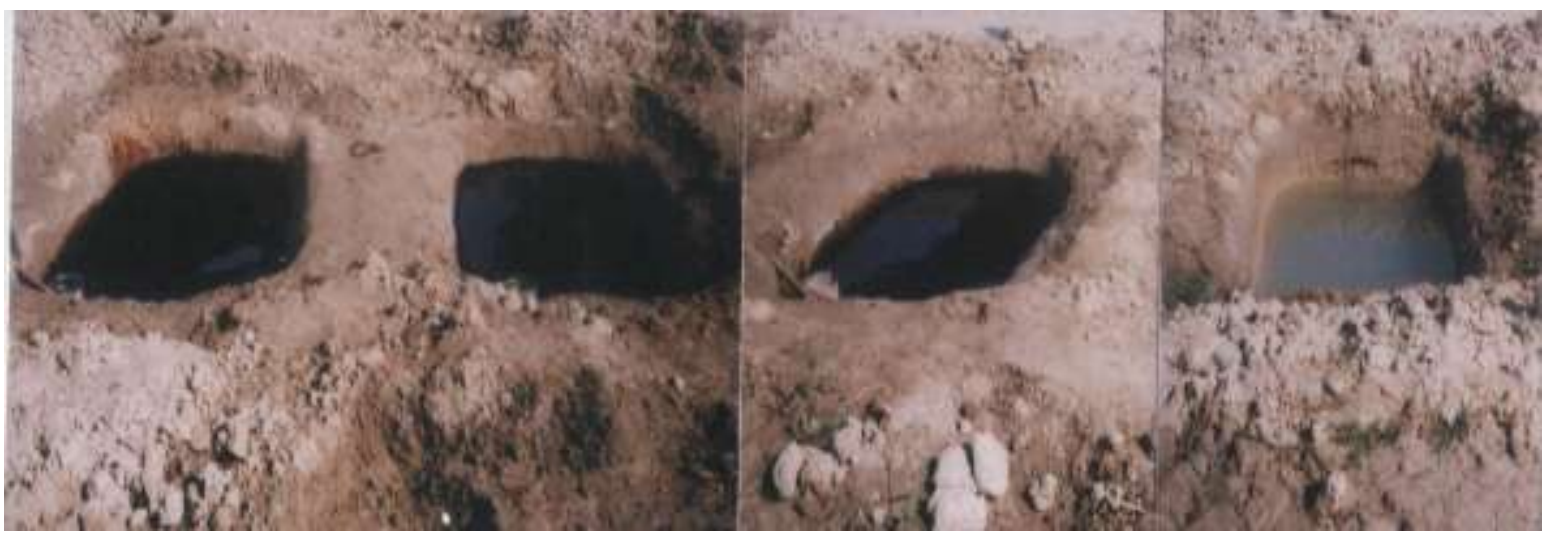

4

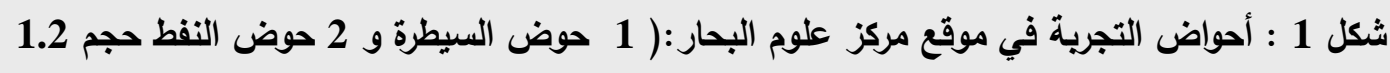

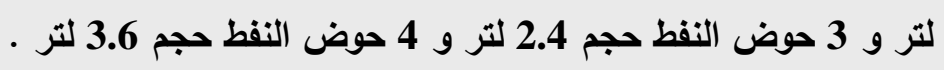

الهثتة ( 7 و 12 و 17 و 22 و 60 يوم )

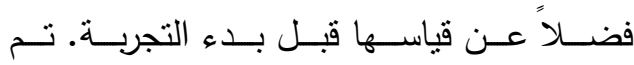

الاستخلاص حسب الطريقة المستخدمة من قبل برنـامج الامم المتحدة لحمايـة البيئة في دليلها لاستخلاص الهيـدروكربونات UNEP (1989)

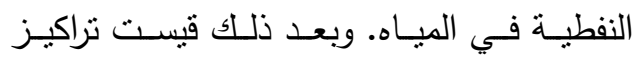

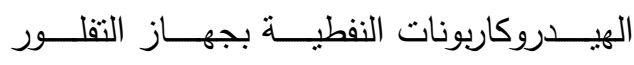

Shimadzu RF نوع Spectrofluorometer

النتائج والمناقثنة

عند حصول تسرب نفطي في البيئة المائية يحدث لطبقة النفط المنسرب تجوية بيئية بفعل ضوء وحرارة الثمس والظروف الجوية من رياح وحركة أمواج إذ تعاني طبقة النفط من تغيرات فيزيائية وكيميائية تنلخص بالثكل 2 .
اجريــت مجموعـة مـن القياســات ليـيـاه الاحواض الاربعة تحت طبقة النفط وذلك بعد مرور فترات زمنية مختلفة (1 ساعة و 7 و 12 و 17 و 22 يوم ) من لحظة اضافة النفط لكل حوض ـ فقد قيست درجة الحرارة بواسطة محرار

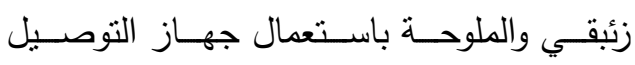
الكهربائي نوع (LF912) ويتم التحويل بالاعتماد على المعادلة التالية: الملوحـة \% \% = التوصـيل الكهربـائي بوحـدة

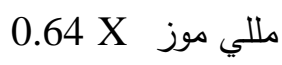
وتم قياس تركيز الاوكسجين المذاب حسب طريقة ونكلر (Lind, 1979). استخلاص الهيدروكاريونات النفطية في الماء تم تقدير تراكيز الهيدروكاربونات النفطية

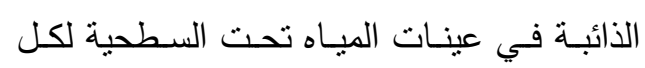
حوض من الاحواض الثلاثة فضلاً عن حوض النان

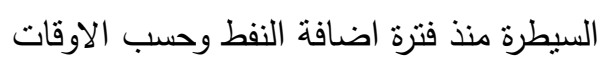




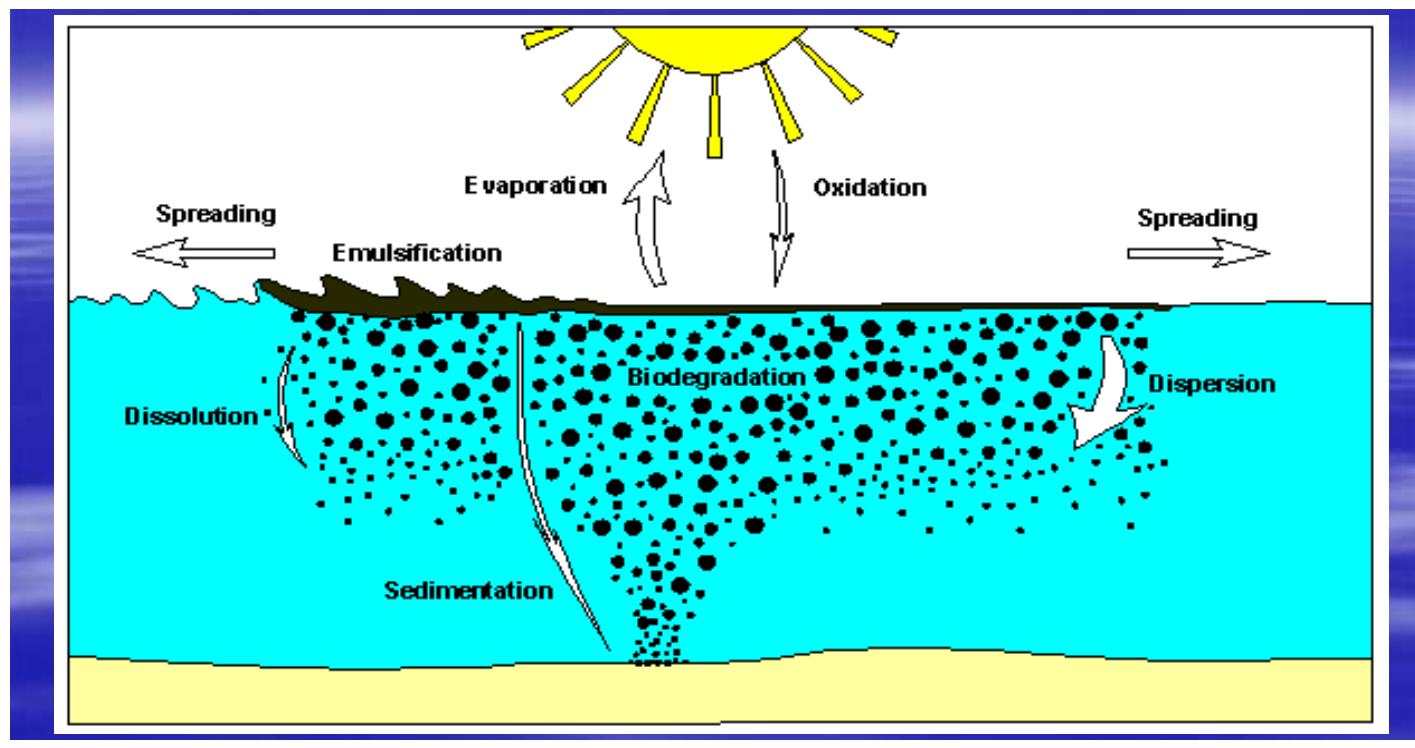

شكل 2 : العمليات التي تحدث لطبقة النفط المتسرب في البيئة المائية نتيجة لظاهرة التجوية.

(Service ) · إن سرعة وأهية أي من عمليات

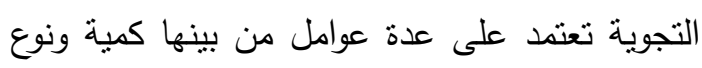

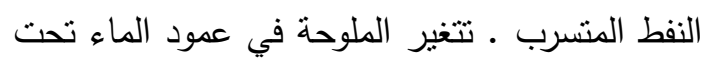

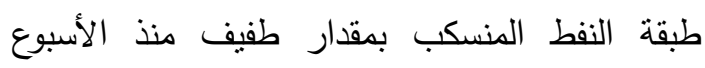

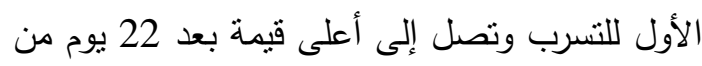

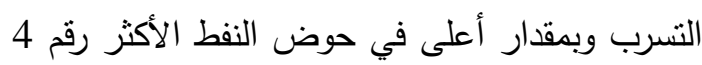
حيث وصلت إلى 2.61 جزء بالألف مقارنة بـ 1.55 جزء بالألف خلا الساعة الأولى للتسرب. إن ملوحة

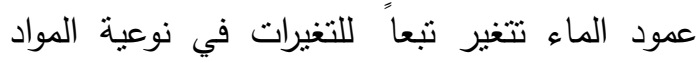

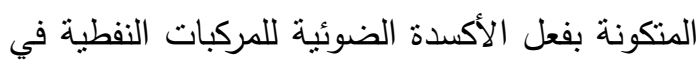

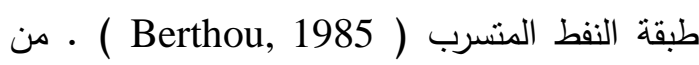
قياسات تركيز الأوكسجين الذائب في أحواض الدراسة

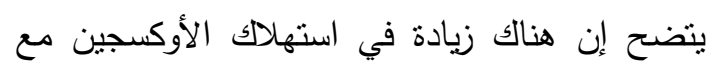

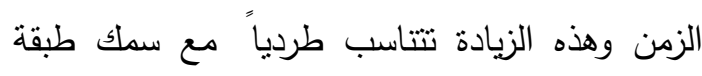
النفط ( Warner et al., 1985 ) ومن الجدول رقم 1 يتضح إن هناك نقصان في تركيز الأوكسجين

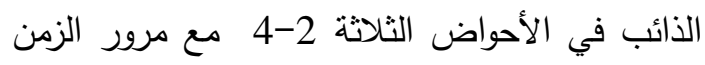
ويتناسب النقصان طردياً مع سمك طبقة النفط في

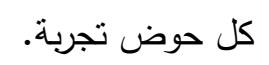

تم قياس المتغيرات الفيزيائية وهي درجة الحرارة والتوصيل الكهربائي (والذي حسبت منه الملوحة) والاوكسجين الذائب حقلياً ( جدول 1 والأثكال 3 ، 4 ، 5 ) · ت تجرى دراسات تجوية النفط لاستقراء التأثيرات والتغيرات الحاصلة في طبقة النفط واحتمالية

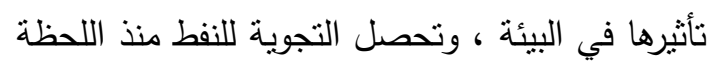

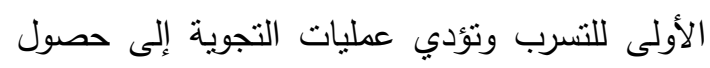
تغيرات في المواصفات الفيزيائية والكيميائية للنفط اللفي المنسرب فضلاً عن حصول تغيرات فيزياتئية وكيميائية في عمود الماء تحت الطبقة المتسربة · .(U.S. Fish \& Wildlife Service, 2007) النفط عبارة عن مزيج من مواد كيمياوية مختلفة بعضاً منها تتصف بالنطاير أسرع من غيرها والتي هوني تتبخر بشكل أسرع عندما ينسكب النفط في البيئة والبعض الآخر يتصف بسهولة التكسير بالأحياء المجهرية وبإمكان أثنعة الثمس أن تكسر مكونات النفط بعملية تعرف بالتكسير الضوئي ويكون حاصل النصل

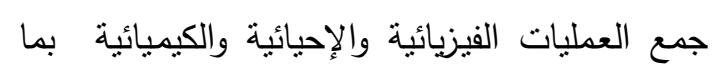

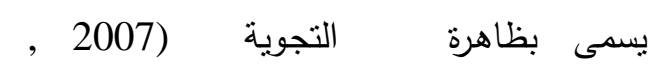
WeatheringNOAA's National Ocean 
جدول 1 : قياسات درجة الحرارة و الملوحة والأوكسجين الذائب في عمود الماء تحت طبقة النفط.

\begin{tabular}{|c|c|c|c|c|}
\hline 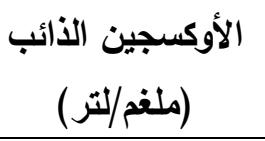 & 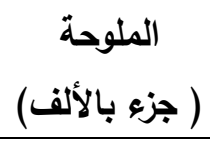 & درجة الحرارة & زمن القراءات & رقم الحوض \\
\hline 8.8 & 1.50 & 9.5 & بعد ساعة & \multirow{5}{*}{ السيطرة 1} \\
\hline 8.9 & 1.51 & 9.3 & بعد 7 يوم & \\
\hline 8.9 & 1.63 & 9.8 & بعد 12 يوم & \\
\hline 8.7 & 1.64 & 9.6 & بعد 17 يوم & \\
\hline 8.9 & 1.70 & 9.9 & بعد 22 يوم & \\
\hline 8.5 & 1.55 & 9.6 & بعد ساعة & \multirow{5}{*}{$\begin{array}{c}2 \\
1.2\end{array}$} \\
\hline 7.1 & 1.74 & 9.9 & بعد 7 يوم & \\
\hline 6.7 & 1.98 & 10.3 & بعد 12 يوم & \\
\hline 6.0 & 2.0 & 10.5 & بعد 17 يوم & \\
\hline 5.0 & 2.12 & 10.5 & بعد 22 يوم & \\
\hline 8.5 & 1.5 & 9.8 & بعد ساعة & \multirow{5}{*}{$\begin{array}{c}3 \\
2.4 \\
\text { لتر نفط }\end{array}$} \\
\hline 6.8 & 2.06 & 10.2 & بعد 7 يوم & \\
\hline 6.0 & 2.04 & 10.8 & بعد 12 يوم & \\
\hline 5.1 & 2.10 & 11.0 & بعد 17 يوم & \\
\hline 4.8 & 2.22 & 11.2 & بعد 22 يوم & \\
\hline 8.0 & 1.57 & 10.0 & بعد ساعة & \multirow{5}{*}{$\begin{array}{c}4 \\
3.6\end{array}$} \\
\hline 5.9 & 2.41 & 10.3 & بعد 7 يوم & \\
\hline 5.1 & 2.34 & 11.1 & بعد 12 يوم & \\
\hline 4.6 & 2.49 & 11.3 & بعد 17 يوم & \\
\hline 4.0 & 2.61 & 11.7 & بعد 22 يوم & \\
\hline
\end{tabular}



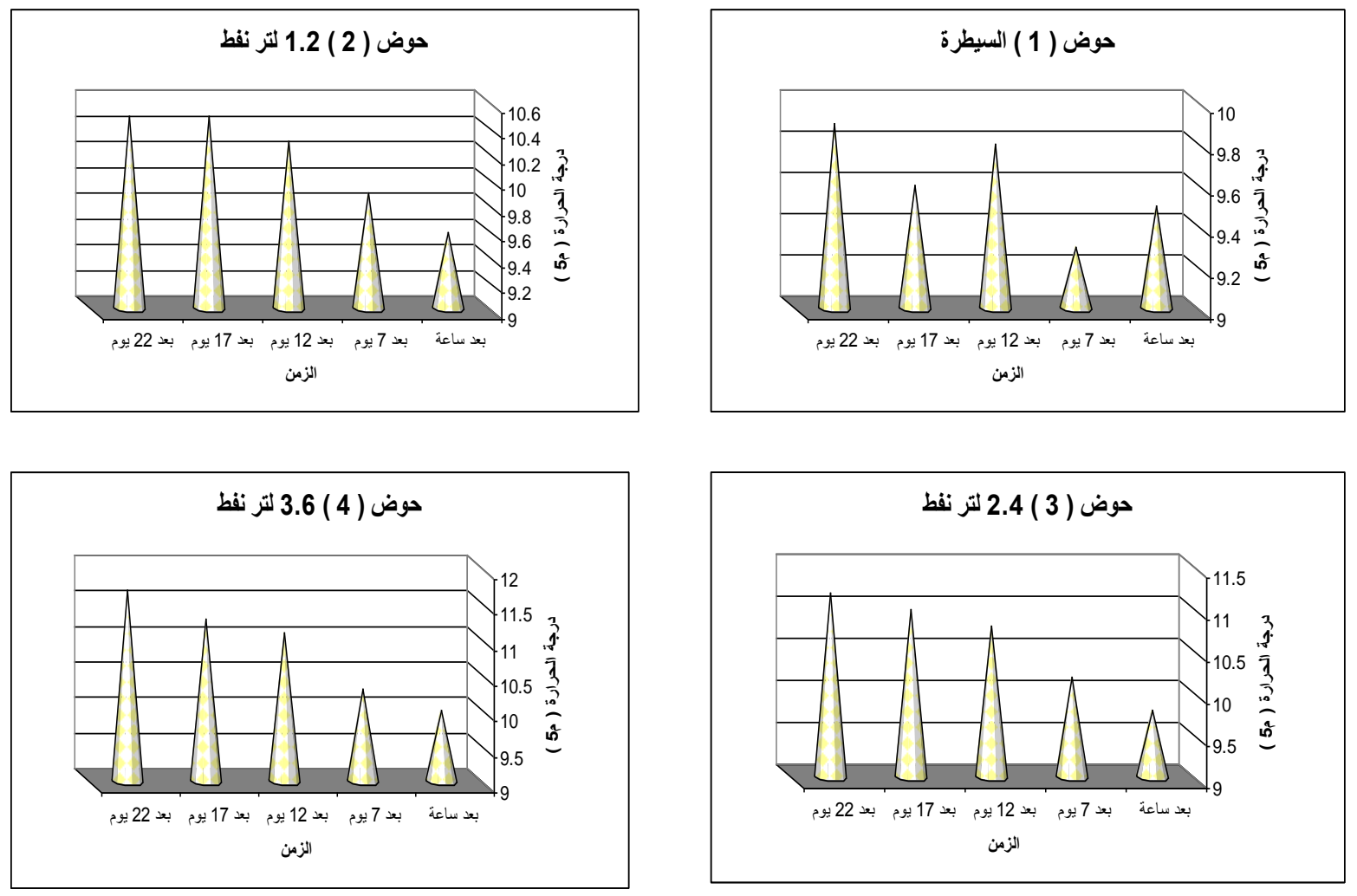

شكل 3 : معدلات درجات الحر ارة ( مº ) في عمود الماء تحت طبقة النفط خلال فترة الدراسة .
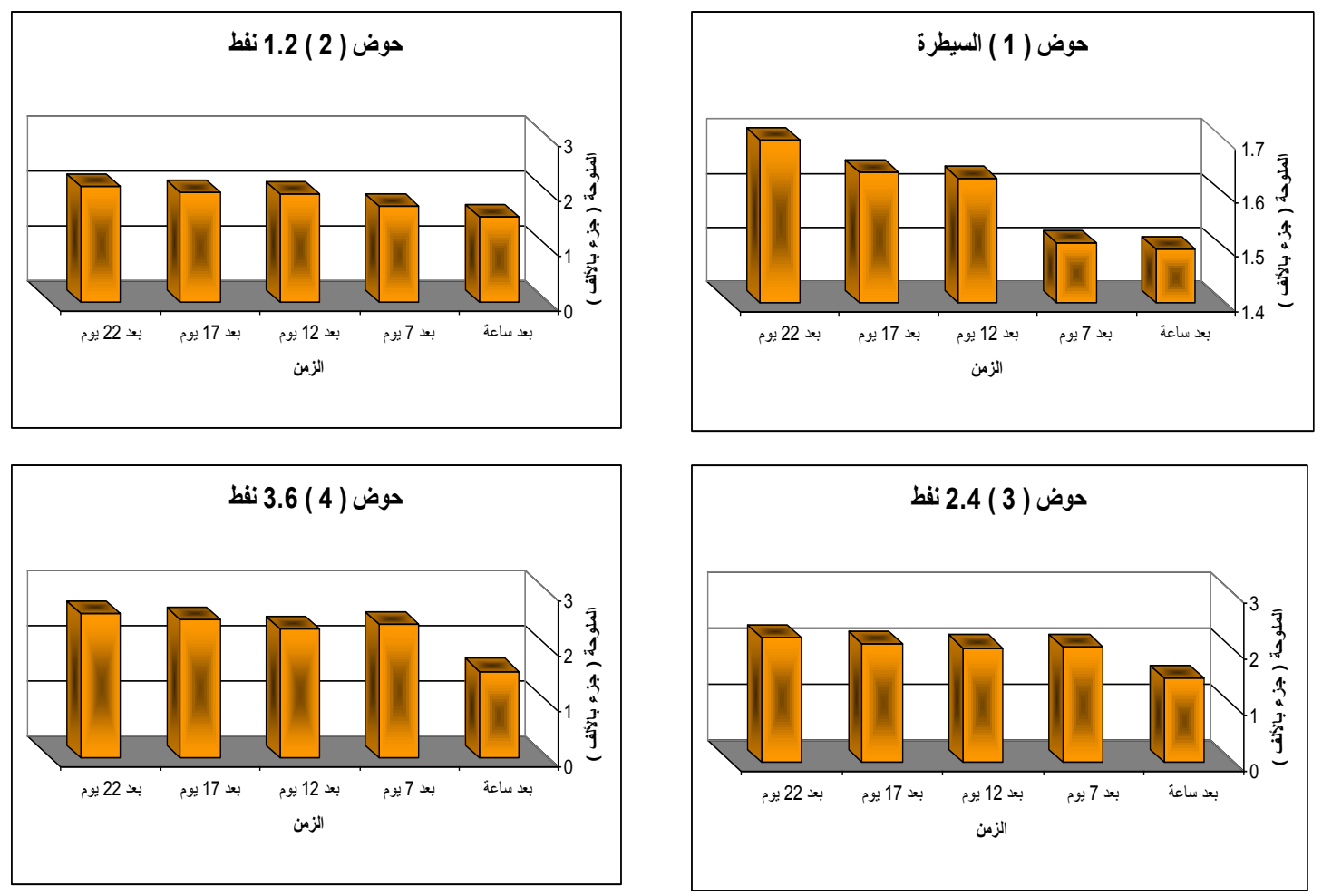

شكل 4 : معدلات الملوحة ( جزء بالألف ) في عمود الماء تحت طبقة النفط خلال فترة الدراسة . 

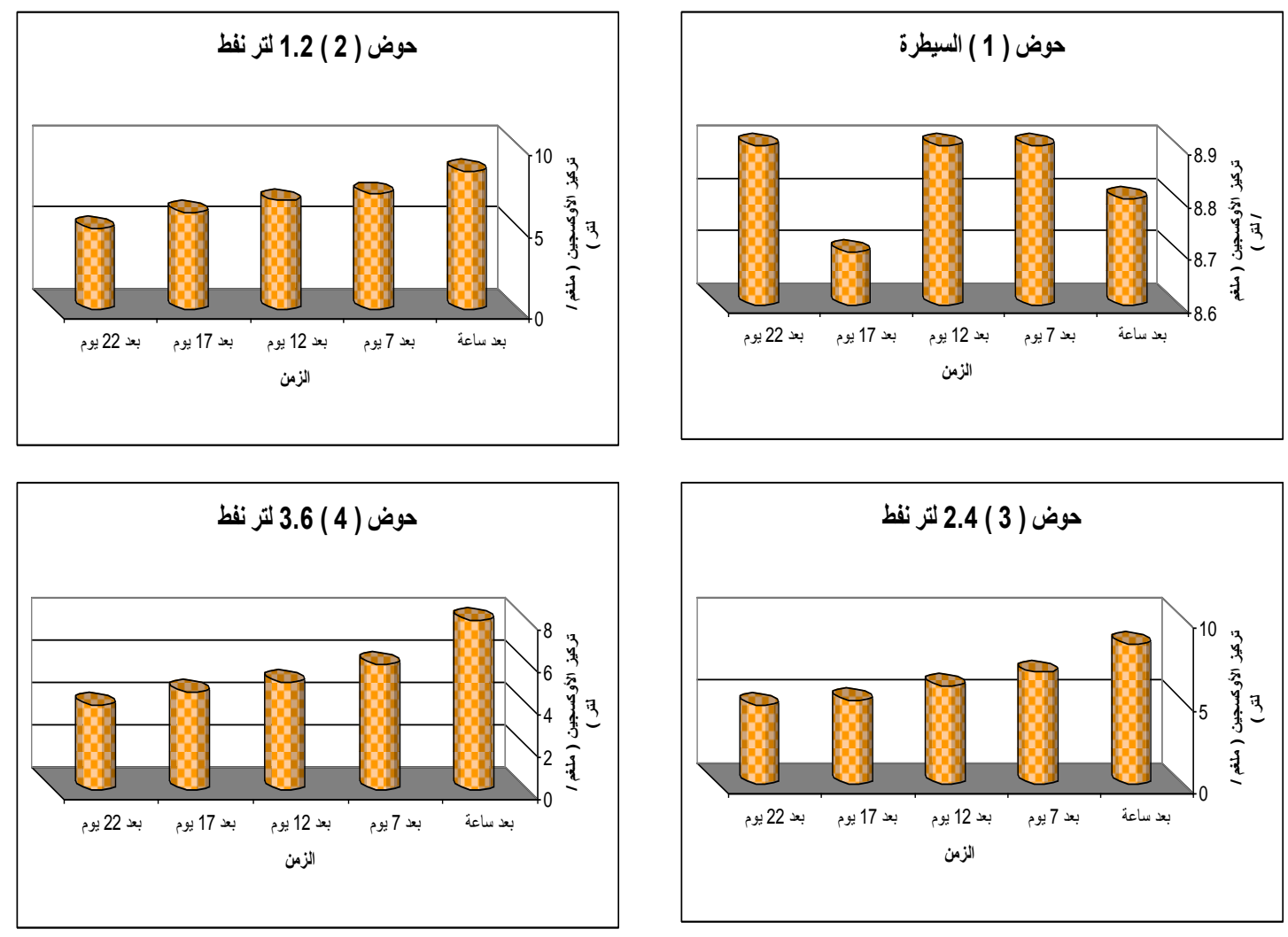

شكل 5: معدلات الأوكسجين الذائب ( ملغم / لتر ) في عمود الماء تحت طبقة النفط خلال فترة الدر اسة.

المـاء المحبط بها ومسن بين المكونـات ذات الذوبانيـة العاليـة في عمـود المـاء المركبـات الهيدروكاربونيـة لئة

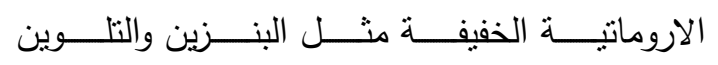
والتي يزداد نركيزها بمرور الزمن (ITOPF,2007)

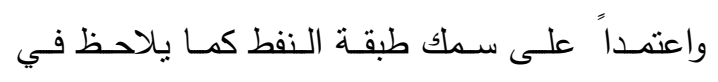

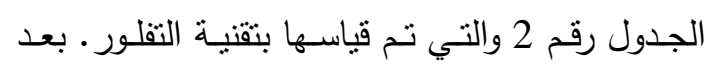

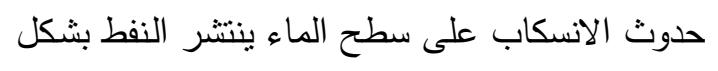

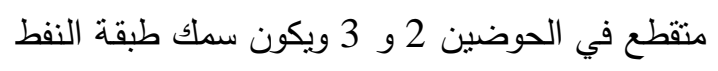

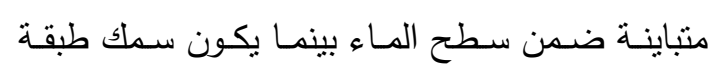
النفط في الحوض رقم 4 مغطية سطح الحوض بشكل

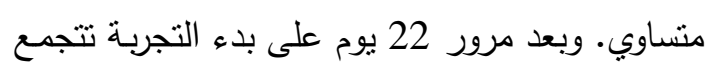

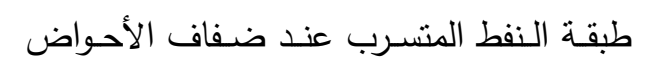

.(Gordon et al., 1976)
تم تقدير الهيدروكاربونات النفية لعينات المياه في

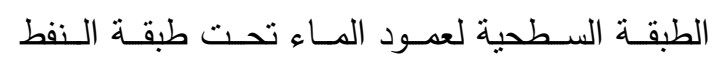
المتسرب, وادرجت النتائج في الجدول 2 والثكل 6 ، 6

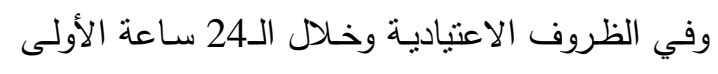

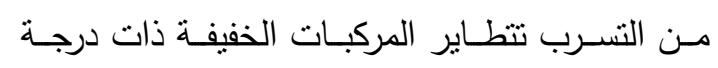

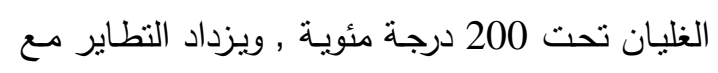
مرور الزمن كلما انتشرت بقعة النفط وأصبح سمكها

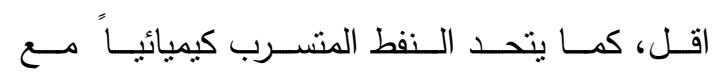
الأوكسـجين والتـي تسـاهم بها أثـعة الثـمس بمقدار

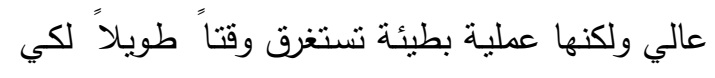
يتحول النفط المتسرب إلى نواتج ذات ذوبانيـة عاليـة تتتقل من طبقة النفط إلى عمود 
يتميز فصل الصيف في مناطق الخليج العربي بنهار طويل ودرجة سطوع شمسي عاليـة. ويعاني

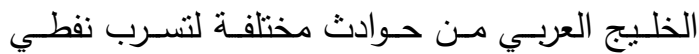
دائسي والذي يؤثر بشكل مبانشر أو غير مباشر في

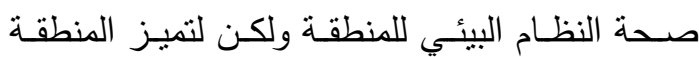
بدرجة سطوع شمسي شديدة وطويلة فإنها نسهم بشكل مباشر في التخلص من الكثير من الهيدروكاربونات

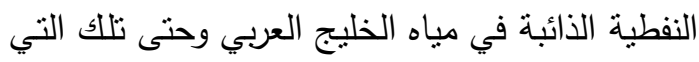
تعـاني مـن تجويـة شـديدة وتتجـرف نحسو الثـــواطئ لتتجمع هناك وتهدد النظام البيئي باللتوث. لذا بت من

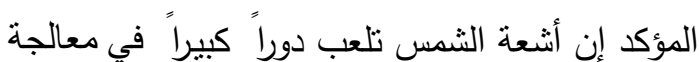

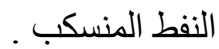
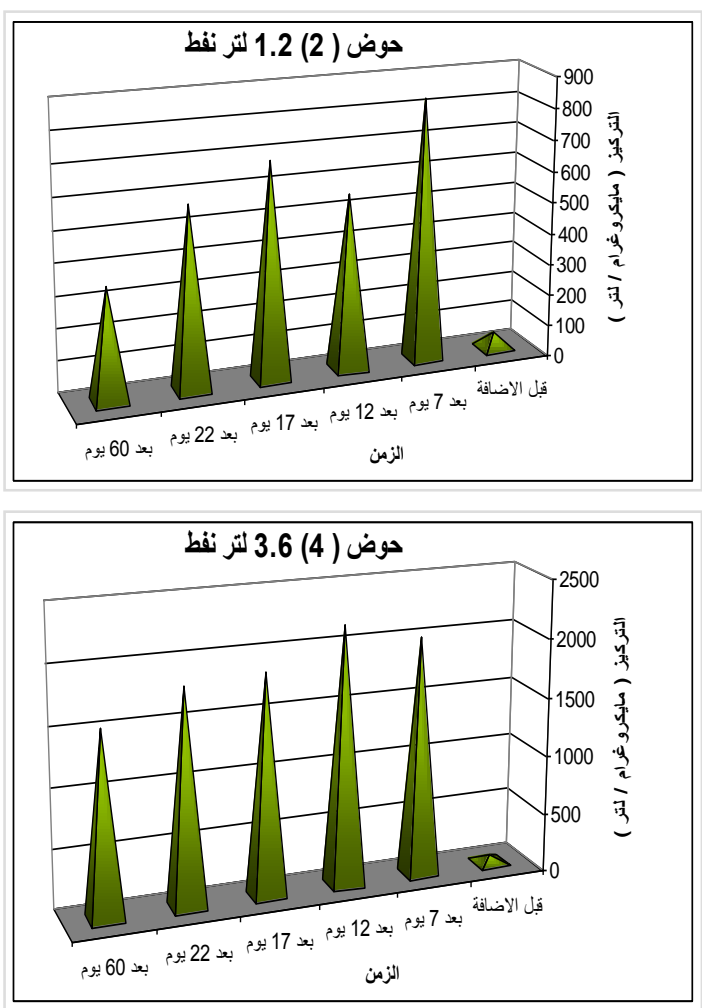

اعتمدت تقنية التفلور الطيفية في تقدير المركبات

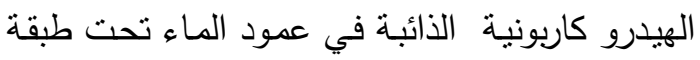
النفط المتسرب وان قسماً من هذه المركبات يتكون فئن

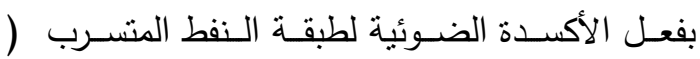
(Berthou, 1985 الهيدروكاربونيـة ضمن طبقة النفط المنسرب تذوب تلقائياً في عمود الماء ومن بين هذه المركبات تلك لكي

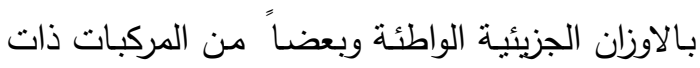
الاوزان الجزيئية العالية مثل الاندينات (Indanes) و النفتالينـات (Naphthalenes) و الاسـينفينيات والفينانثرينــــــ (Acenaphthenes) (McAullffe, 1969; , (Phenanthrenes) . Lysyi and Ressell, 1974)
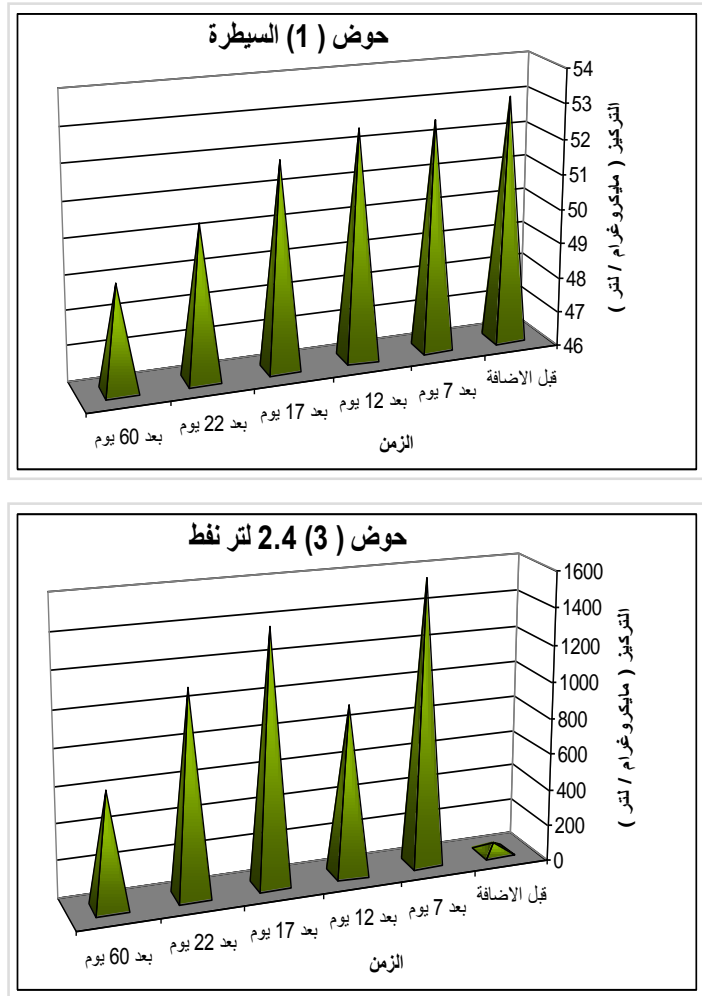

شكل 6 : معدلات تراكيز الهيدروكاربونات النفطية ( مايكروغرام / لتر ) في عمود الماء تحت طبقة النفط خلال فترة الدراسة. 
جدول 2 : قيم نراكيز الهيدروكاربونات النفطية في عمود الماء تحت طبقة النفط ( مايكروغراج/ لتر).

\begin{tabular}{|c|c|c|c|}
\hline SD & التركيز & زمن القراءات & رقم الحوض \\
\hline 2.40 & 53.04 & قبل الإضافة & \multirow{6}{*}{$\begin{array}{c}1 \\
\text { السبطرة }\end{array}$} \\
\hline 2.31 & 52.56 & بعد 7 يوم & \\
\hline 2.40 & 52.56 & بعد 12 يوم & \\
\hline 2.22 & 51.89 & بعد 17 يوم & \\
\hline 2.30 & $\mathbf{5 0 . 3 7}$ & بعد 22 يوم & \\
\hline 2.21 & 48.98 & بعد 60 يوم & \\
\hline 2.40 & 53.04 & قبل الإضافة & \multirow{6}{*}{$\begin{array}{c}2 \\
1.2 \text { لتر نفط }\end{array}$} \\
\hline 1.99 & 833.96 & بعد 7 يوم & \\
\hline 3.25 & 556.21 & بعد 12 يوم & \\
\hline 4.40 & 685.66 & بعد 17 يوم & \\
\hline 2.91 & 577.98 & بعد 22 يوم & \\
\hline 3.01 & 355.22 & بعد 60 يوم & \\
\hline 2.60 & 54.30 & قبل الإضافة & \multirow{6}{*}{$\begin{array}{c}3 \\
2.4 \text { لتر نفط }\end{array}$} \\
\hline 8.12 & 1564.33 & بعد 7 يوم & \\
\hline 7.91 & 929.91 & بعد 12 يوم & \\
\hline 6.92 & 1382.67 & بعد 17 يوم & \\
\hline 5.02 & 1112.23 & بعد 22 يوم & \\
\hline 2.09 & 623.65 & بعد 60 يوم & \\
\hline 3.02 & $\mathbf{5 5 . 8 0}$ & قبل الإضافة & \multirow{6}{*}{$\begin{array}{c}4 \\
3.6\end{array}$} \\
\hline 15.51 & 2031.48 & بعد 7 يوم & \\
\hline 16.22 & 2189.32 & بعد 12 يوم & \\
\hline 10.91 & 1868.35 & بعد 17 يوم & \\
\hline 9.12 & 1811.52 & بعد 22 يوم & \\
\hline 8.05 & 1549.21 & بعد 60 يوم & \\
\hline
\end{tabular}


Mackay et al., 1981. Oil Weathering Test, Mackey Apparatues :Oil Spill Laboratory

McAullffe, C. D. 1969. Determination of dissolved hydrocarbons in subsurfacebrines. Chem. Geol. 4:225-233.

Mikhilova, L. V., 1987. Characteristics of the behaviour of the watersoluble fraction of oil in model experiments. Water Res., 13:197205.

NOAA's National Ocean Service. 2007. Fingerprinting of oil.

NAS, 1985. Oil in the sea: Inputs, fate and effects. National Academy Press, Washington, DC, 1985.

Payne, J. R. and Phillips, C. R. 1985. Photochemistry of petroleum in water. Environ. Sci. Tech., 19:569-579.

Stiver, W., Shiu, W. Y. and Mackay, D., 1989. Evaporation times and rates of specific hydrocarbons oil spill. Environ. Sci. Tech., 23:101-105

UNEP (United Nations Environmental Programme) (1989). Comparative Toxicity Test of Water Accommodated Fraction of Oil Dispersants to Marine Organism . Reference Methods For Marine pollution . No, 45, 21p.

U.S. Fish \& Wildlife Service, 2007. Effect of oil spill on wildlife and habitat. http//www.fws.gov

Warner, M. D., Adams, V. D., Lamarra, V. A. and Winters, N. L. 1985. Responses of model freshwater ecosystem to crude oil . Water Res., 19:285-292.

\section{References}

Berthou, F., Ducreeux, J. and Bodennec, G., 1985. Analysis of watersoluble acid compounds derived from spilled oil in a controlled marine enclosure. Intern, J. Environ. Anal. Chem., 21:267282.

Blumer, M., Erhardt, M. and Jones, J. H. 1973. The environmental fate of stranded crude oil . Deep-Sea, 20:239-259.

DouAbul, A. Az. and Al-Saadi, M. K. 1990. Oil spills in riversweathering of stranded crude. Marine Mesopotamica, 5:293303.

Gordon, D. C. Jr., Kelzer, P. D., Hardstaff, W. R. and Aldous, D. G. 1976. Fate of crude oil spilled on seawater contained in outdoor tanks. Environ. Sci. Tech., 10:280-285.

Hanson, H. P. 1975. Petrochemical degradation of petroleum hydrocarbon surface films on seawater. Mar. Chem., 3:183-195.

ITOPF, 2007. Technical Information Paper No.2: Fate of Marine Oil Spills.

Kennicutt, M. C. 1988. The effect of biodegradation on crude oil bulk and molecular composition. Oil and Chem. Poll., 4:89-112

Lee, R. F., Gardner, W. S., Anderson, J. W., Blaylock, J. W. and BarwellClarke, J. 1978. Fate of polycyclic aromatic hydrocarbons in controlled ecosystem enclosures. Environ. Sci. Tech., 12:832-838.

Lind, O. T. 1979. Handbook of Common Methods in Limnology. The C.V. Mos by Company London $199 \mathrm{p}$.

Lysyi, I. and Ressell, E. C. 1974. Dissolution of petroleum derived products in water. Water Res. 8:863-868. 


\title{
Field Study for the Effect of Spilled Crude Oil upon some Environmental Parameters of Water and Their Estimation
}

\author{
Faris J. M. Al-Imarah Ali M. Nasir \\ Dept. Marine Environm. Chemistry - Marine Science Centre \\ Basrah University \\ Basrah - Iraq.
}

\begin{abstract}
$\underline{\text { Abstract }}$
A study has been conducted for spilled crude oil in sand reservoir $2 \times 2 \times 1 \mathrm{~m}^{3}$ each contains 12001 water from Garmat Ali River, crude oil was poured in each reservoir as follows: 1.2, 2.4 and 3.61 in the first three reservoirs respectively while the fourth was left without oil as control. The four reservoir were exposed to climate under the sun and wind, the changes in the spilled oil layers were investigated as well as some environmental parameters for water underneath which are temperature, salinity and dissolved oxygen. Moreover, petroleum hydrocarbons were measured after certain intervals of 7, 12, 22 and 60 days of exposure. Spilled oil layers reduced with time as petroleum hydrocarbons dissolved in the water for reservoir with oil in which concentrations of dissolved petroleum hydrocarbons increased directly with thickness of oil spill compared with control reservoir. After a week of exposure dissolved petroleum hydrocarbons measured were $833.96,1564.33$ and $2031.48 \mu \mathrm{g} / \mathrm{l}$ in the reservoir 1,2 and 3 respectively compared with $53.04 \mu \mathrm{g} / \mathrm{l}$ in the control reservoir. These levels were decreased with time in which they reached to $355.22,623.65$ and $1549.21 \mu \mathrm{g} / \mathrm{l}$ in the reservoirs 2,3 and 4 respectively after 60 days of exposure. Results were explained on the basis of biodegradation, photo-oxidation due to increase temperature and decrease dissolved oxygen accompanied the exposure, in which temperature increased directly while dissolved oxygen decreased with exposure time and thickness of spilled oil. More over, salinity is increased with time to be 1.57, 2.41, 2.34, 2.49 and $2.61 \%$ during exposure times 1 hour, 7, 12, 17 and 22 days respectively in the reservoir No. 4. As thickness of oil slick increased the salinity is increased to become 2.12, 2.22 and $2.61 \%$ o in the reservoirs 2,3 and 4 respectively after 22 days of exposure to natural conditions.
\end{abstract}

